\title{
Extracción de nutrientes minerales en hojas y frutos de ají (Capsicum sp.), y su influencia en el rendimiento
}

\section{Extraction of mineral nutrients in pepper (Capsicum sp.) leaves and fruits and its influence on yield}

MARÍA DEL PILAR ROMERO-LOZADA ${ }^{1}$

YINA JAZBLEIDI PUENTES-PÁRAMO2,3

JUAN CARLOS MENJIVAR-FLORES ${ }^{2}$

Ají tipo Habanero (Capsicum

chinense J.) en producción.

Foto: J.C. Menjivar-Flores

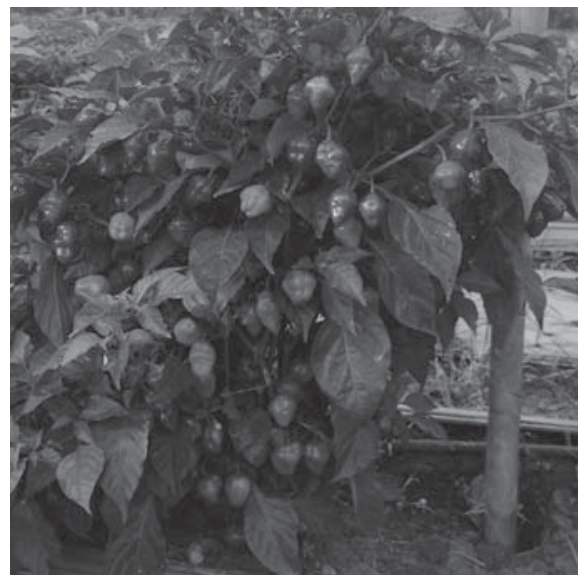

\section{RESUMEN}

La investigación tuvo como objetivo determinar la extracción y distribución de nitrógeno (N), fósforo (P) y potasio (K) en hojas y frutos, de dos especies de ají: Capsicum frutescens L. (tipo Tabasco) y Capsicum chinense J. (tipo Habanero), y su influencia en el rendimiento. El diseño incluyó los cuatro tratamientos: Testigo, programa de fertilización que maneja el Centro Experimental de la Universidad Nacional de Colombia, sede Palmira (CEUNP) (N: 150, $\mathrm{P}_{2} \mathrm{O}_{5}: 100$ y $\left.\mathrm{K}_{2} \mathrm{O}: 300\right)$, CEUNP + 50\% y CEUNP + 100\% de N-P-K. El análisis de varianza mostró diferencias altamente significativas $(P \leq 0,01)$ en la concentración de nutrientes N-P-K en fruto y hoja para los dos tipos de ají; así, la mayor concentración de N se encontró en hojas y de K en frutos, coincidiendo con menor concentración de P para hojas y frutos. Habanero presentó el mayor rendimiento $(23.807$ kg ha-1), como también, la mayor extracción de N-P-K por tonelada de fruto fresco. Ambas especies de ají guardan la misma preferencia de concentración de nutrientes en hoja: $\mathrm{N}>\mathrm{K}>\mathrm{P}$ y fruto: $\mathrm{K}>\mathrm{N}>\mathrm{P}$, sin embargo, es evidente la diferencia que existe en concentración y extracción de nutrientes, como también en el rendimiento, lo cual sugiere la influencia del genotipo y por tanto, conveniente realizar un diagnóstico y manejo nutricional específico para cada especie de ají.

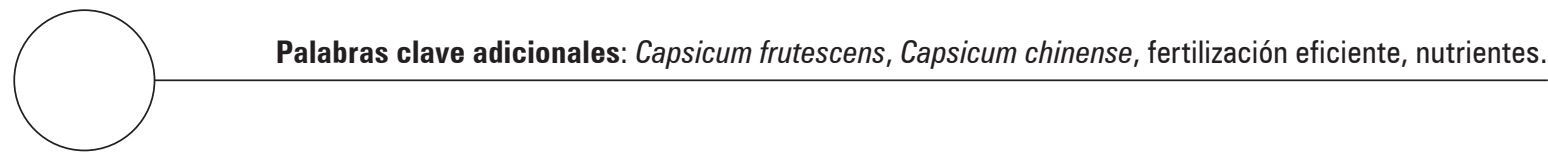

\footnotetext{
1 Facultad de Ciencias Agropecuarias, Programa de Maestría en Ciencias Agrarias, Universidad Nacional de Colombia, Palmira (Colombia).

2 Facultad de Ciencias Agropecuarias, Universidad Nacional de Colombia, Palmira (Colombia).

3 Autor para correspondencia. yjpuentesp@unal.edu.co
} 


\section{ABSTRACT}

This research aimed to determine the extraction and distribution of nitrogen $(\mathrm{N})$, phosphorus $(\mathrm{P})$ and potassium $(\mathrm{K})$ in leaves and fruits, for two species of pepper: Capsicum frutescens L. (type Tabasco) and Capsicum chinense J. (type Habanero), and their influence on yield. The experiment design included four treatments: Control, fertilization program of the Experimental Center of the National University of Colombia, located in Palmira (CEUNP) (N: 150, $\mathrm{P}_{2} \mathrm{O}_{5}: 100$ and $\mathrm{K}_{2} \mathrm{O}: 300$ ), CEUNP $+50 \%$ and CEUNP $+100 \%$ of N-P-K. The analysis of variance showed highly significant differences $(P \leq 0.01)$ in the nutrient N-P-K concentration in the fruits and leaves for the two species of pepper. The highest concentration of $\mathrm{N}$ was found in the leaves and the highest $\mathrm{K}$ in the fruits, coinciding with the lowest concentration of $\mathrm{P}$ in the leaves and fruits. Habanero presented the highest yield $\left(23,807 \mathrm{~kg} \mathrm{ha}^{-1}\right)$, as well as the highest N-P-K extraction per ton of fresh fruit. Both types of pepper had the same preference for nutrient concentration in the leaves: $\mathrm{N}>\mathrm{K}>\mathrm{P}$ and fruit: $\mathrm{K}>\mathrm{N}>\mathrm{P}$; however, a difference was evident in the concentration and extraction of nutrients, as well as in the yield, suggesting the influence of the genotype. Therefore, a specific nutritional diagnosis and management for each species of pepper would be appropriate.

Additional key words: Capsicum frutescens, Capsicum chinense J., efficient fertilization, nutrients.

Fecha de recepción: 05-02-2017 Aprobado para publicación: 15-04-2017

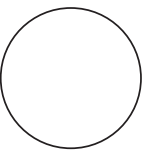

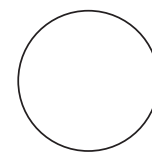

El ají (Capsicum spp.) es una hortaliza del género Capsicum (solanáceas) que posee cinco especies domesticadas, incluyendo C. frutescens L. (tipo Tabasco) y C. chinense J. (tipo Habanero) (Bosland y Votava, 2012). Estos materiales se caracterizan por presentar frutos pequeños de diferente forma, sabor picante y ser de uso condimentario (Vallejo y Estrada, 2004).

Los cultivos hortícolas presentan limitantes que reducen su potencial productivo, en este sentido, una nutrición balanceada permite obtener el máximo rendimiento (Nieves et al., 2015). Marschner (1995) menciona que la disponibilidad, absorción y distribución de nutrientes esenciales en la planta son factores que ejercen mayor efecto sobre el crecimiento y rendimiento de los cultivos, en tanto, es claro que la absorción de nutrientes depende del tipo de cultivo, edad, disponibilidad de nutrientes y agua (Salisbury y Ross, 2000).

Por otra parte, Quintal et al. (2012) encontraron un incremento del rendimiento en ají $C$. chinense con una lámina de agua correspondiente al $60 \%$ de la humedad aprovechable del suelo; Zayed (2013) menciona que la productividad de ají $C$. annuum se puede ver afectada por el contenido y calidad de los fertilizantes orgánicos, o por una limitada disponibilidad y absorción de nutrientes (Reyes et al., 2014). Así mismo, Alejo et al. (2015) encuentran asociación entre alto contenido de
$\mathrm{N}$ en frutos y mayor rendimiento, lo que evidencia la influencia nutricional sobre el rendimiento.

Dependiendo de la oferta ambiental, la extracción y distribución de nutrientes varia de un cultivo a otro y dentro del mismo cultivo entre cultivares, tipos y órganos de la planta de ají (Salazar y Juárez, 2013); lo mismo sucede con respecto al orden de preferencia de elementos esenciales en los órganos de la planta, encontrándose diferente concentración en hojas e influencia sobre el rendimiento como lo sugiere Puentes et al. (2016); en tanto, el cultivo de ají no es la excepción, Noh et al. (2010) reportan para ají Habanero C. chinense un orden en la concentración de macronutrientes de $\mathrm{N}>\mathrm{K}>\mathrm{Ca}>\mathrm{Mg}$ y $\mathrm{P}$ y para micronutrientes $\mathrm{Fe}>\mathrm{Zn}>\mathrm{Mn}$ y $\mathrm{Cu}$, desde la etapa de desarrollo correspondiente a $50 \mathrm{~d}$ después del trasplante hasta cosecha, $120 \mathrm{~d}$.

Conocer la extracción, distribución y tendencia en el contenido de nutrientes para frutos y hojas en función de la dosis de fertilización aplicada, es importante para predecir una disminución del rendimiento por un desbalance nutricional, en especies diferentes como C. chinense y C. frustescens. Sin embargo, en la Universidad Nacional de Colombia sede Palmira con una colección de 770 accesiones de ají (Pardey et al., 2009), solamente han sido evaluadas con base al contenido de capsaicina, tolerancia a plagas y 
enfermedades, y algunos trabajos de investigación en fertilización.

Así, la importancia de evaluar cada material de ají con el fin de hacer un apropiado manejo nutricional y determinar su influencia en el rendimiento en respuesta a diferentes niveles de fertilidad en el suelo, la extracción en fruto y distribución de nutrientes en hoja y fruto para las especies C. chinense y C. frutescens es el objetivo de esta investigación, ya que en la actualidad reciben el mismo manejo nutricional, siendo especies diferentes.

\section{MATERIALES Y MÉTODOS}

Esta investigación se realizó en el Centro Experimental de la Universidad Nacional de Colombia (CEU$\mathrm{NP}$ ), ubicado geográficamente a $3^{\circ} 24^{\prime} \mathrm{N}$ y $76^{\circ} 26^{\prime} \mathrm{W}$, a $980 \mathrm{msnm}$, en el municipio de Candelaria (vereda El Carmelo) del departamento del Valle del Cauca. La zona presenta una precipitación promedio anual de $1.135 \mathrm{~mm}$, temperatura media del aire de $24^{\circ} \mathrm{C}$ y brillo solar promedio de 172,6 h/mes.

El suelo experimental se muestreo de $20-30 \mathrm{~cm}$ de profundidad para evaluar las características físico-químicas, cuyo análisis fue realizado en el Centro de Agricultura Tropical (CIAT) e interpretado con valores referencia del ICA (1992), mostrando condiciones óptimas para el cultivo de ají. En tanto, el suelo presenta textura arcillosa (arena $24,96 \%$, arcilla $41,04 \%$ y limo $34 \%$ ), densidad aparente $1,2 \mathrm{Mg} \mathrm{m}^{-3}$, capacidad de campo $40,13 \%$, punto de marchitez permanente $18,13 \%$ y porosidad total de $54,54 \%$; además, $\mathrm{pH}$ de 7,7, capacidad de intercambio catiónico $20,95 \mathrm{cmol}$ $\mathrm{kg}^{-1}$, materia orgánica $2,1 \%$, fósforo $75 \mathrm{ppm}$ y potasio $0,42 \mathrm{cmol} \mathrm{kg}^{-1}$.

Los tratamientos fueron diseñados tomando como referencia la dosis utilizada en CEUNP para el cultivo de ají, la cual coincide con el manejo nutricional de los agricultores en diferentes zonas del país, éstos fueron: sin fertilización o testigo (T1), programa de fertilización del CEUNP (N: 150, $\mathrm{P}_{2} \mathrm{O}_{5}: 100$ y $\mathrm{K}_{2} \mathrm{O}: 300$ ) (T2), incrementos del 50 y $100 \%$ de N-P-K sobre el nivel del programa de fertilización del CEUNP, T3 y T4, respectivamente (Romero et al., 2016). Las fuentes de fertilización utilizadas: DAP (18-46-00), Urea (46-0000) y KCl (00-00-60).

Se utilizó un diseño experimental en bloques completos al azar con cuatro tratamientos y cuatro réplicas. Las plántulas fueron obtenidas a partir de la siembra de semillas en condiciones controladas de invernadero, posteriormente, fueron trasplantadas en campo y sembradas a $0,6 \mathrm{~m}$ entre plantas y 1,3 m entre surcos, con densidad de 12.820 plantas/ha. La unidad experimental (UE) utilizada consistió en parcelas de 5,2×9 $\mathrm{m}$, para un total de 16 UE. Las plantas en campo estuvieron sometidas a plena exposición solar, y fueron regadas de acuerdo al balance hídrico.

Se evaluaron dos líneas elite de ají con alta concentración de capsaicina Capsicum frutescens L. tipo Tabasco y Capsicum chinense J. tipo Habanero. Las dosis de fertilización por tratamiento empleadas de acuerdo a las cuatro etapas del cultivo (Noh et al., 2010) fueron: 10 días después del transplante (DDT) 10\%:30\%:10\% correspondiente para N-P-K, 25 DDT: 20\%:25\%:15\%, 45 DDT: 30\%:20\%:20\%, estos tres fraccionamientos anteceden la fase I de desarrollo, 70 DDT: 20\%:150\%:20\%, antecede la fase II de floración y fructificación, 85 DDT: 10\%:10\%:20\% y 90 DDT: 10\%:0\%:15\%, estas dos últimos anteceden la fase III y IV de maduración y cosecha del fruto respectivamente.

La concentración en hoja y fruto se determinó para $\mathrm{N}$ por el método Kjheldhal; $\mathrm{Ca}^{2+}, \mathrm{Mg}^{2+}, \mathrm{K}+, \mathrm{Fe}^{2+}, \mathrm{Mn}^{2+}$, $\mathrm{Cu}^{2+}, \mathrm{Zn}^{2+}, \mathrm{Na}^{+}, \mathrm{B}$ en base seca $\left(550^{\circ} \mathrm{C}\right)$ y absorción atómica; $\mathrm{S}$ por digestión ácida y turbidímetria; P por descomposición en base seca $\left(550^{\circ} \mathrm{C}\right)$ y colorimetría, los cuales fueron realizados en el Centro Internacional de Agricultura tropical (CIAT) siguiendo los protocolos estándares para tejido vegetal (Mckean, 1993). Para los análisis se colectaron 40 hojas recién maduras de a planta en floración (Malavolta, 1997), y 20 frutos maduros de la parte media de la planta; este procedimiento se realizó por cada tratamiento y tipo de material.

El rendimiento se consideró como el peso fresco de frutos cosechados de cada línea y tratamiento, provenientes de 11 cosechas. La extracción de los nutrientes N-P-K se determinó para una tonelada de fruto fresco por cada tipo de material en el tratamiento de mayor rendimiento.

Los datos fueron analizados con el programa Statistical Analysis System (SAS). Mediante análisis de varianza (ANDEVA) se determinaron diferencias entre las variables de respuesta teniendo como fuente de variaciones, el tipo de material, tratamientos y la interacción tipo de material $\mathrm{x}$ tratamiento; posteriormente se empleó la prueba de Tukey $(P \leq 0,05)$ para encontrar diferencias significativas entre los tratamientos. 


\section{RESULTADOS Y DISCUSIÓN}

\section{Concentración de nitrógeno, fósforo y potasio en hoja y fruto}

La concentración de nutrientes muestra diferencias altamente significativas $(P \leq 0,01)$ por tipo de ají en relación al contenido de $\mathrm{N}$ en hojas $(\mathrm{H})$-frutos $(\mathrm{F})$, y para $\mathrm{K}$ en frutos, en contraste, en hojas no hubo diferencias significativas. P no mostró diferencias significativas $(P>0,05)$ para ambos órganos. Con respecto a los tratamientos hubo diferencias $(P \leq 0,01)$ para $N$ en $\mathrm{H}-\mathrm{F}$, significativas para $\mathrm{K}$ en $\mathrm{H}-\mathrm{F}$ y para $\mathrm{P}$ en fruto, más no mostro diferencias en hoja. Mientras que la interacción tipo $\mathrm{x}$ tratamiento no fue significativa $(P>0,05)$ para $\mathrm{P}, \mathrm{K}$ en $\mathrm{H}-\mathrm{F}$, y para $\mathrm{N}$ en fruto, solamente la concentración de $\mathrm{N}$ en hojas fue altamente significativa en la interacción (tabla 1).

Tabla 1. Análisis de varianza en hoja (H) y fruto (F) para dos especies de ají.

\begin{tabular}{|l|c|c|c|c|c|c|}
\hline \multirow{2}{*}{$\begin{array}{c}\text { Fuente de } \\
\text { variación }\end{array}$} & \multicolumn{2}{c|}{ N } & \multicolumn{2}{c|}{ P } & \multicolumn{2}{c|}{ K } \\
\cline { 2 - 7 } & H & F & H & F & H & F \\
\hline Tipo & $* *$ & $* *$ & NS & NS & NS & $* *$ \\
\hline Tratamiento & $* *$ & $* *$ & NS & $*$ & $*$ & $*$ \\
\hline Tipo x Tratamiento & $* *$ & NS & NS & NS & NS & NS \\
\hline
\end{tabular}

**: diferencias altamente significativas $(P \leq 0,01)$; *: diferencia significativa $(P \leq 0,05)$; NS: diferencia no significativa $(P>0,05)$.

En el tipo Habanero la mayor concentración de $\mathrm{N}$ en hoja se encontró en el tratamiento T3 $\left(48,15 \mathrm{~g} \mathrm{~kg}^{-1}\right)$ y la menor en T1 $\left(42,11 \mathrm{~g} \mathrm{~kg}^{-1}\right)$; en fruto, la mayor concentración en T4 $\left(27,09 \mathrm{~g} \mathrm{~kg}^{-1}\right)$ y menor en T1 $(22,38$ $\mathrm{g} \mathrm{kg}^{-1}$ ). Resultados similares obtuvo Alejo et al. (2015) quienes sugieren que a mayor dosis de $\mathrm{N}$ mayor concentración de este nutriente en frutos. La mayor concentración de P en hojas fue para el T2 $\left(4,69 \mathrm{~g} \mathrm{~kg}^{-1}\right)$ y la menor en T1 $\left(4,46 \mathrm{~g} \mathrm{~kg}^{-1}\right)$, y en fruto, en T3 $(3,20 \mathrm{~g}$ $\left.\mathrm{kg}^{-1}\right)$ y T1 $\left(2,96 \mathrm{~g} \mathrm{~kg}^{-1}\right)$.

Para K la mayor concentración en hoja se obtuvo en T4 $\left(41,18 \mathrm{~g} \mathrm{~kg}^{-1}\right)$ y T1 $\left(28,91 \mathrm{~g} \mathrm{~kg}^{-1}\right)$; en fruto, en T3 $\left(30,82 \mathrm{~g} \mathrm{~kg}^{-1}\right)$ y la menor en T1 $\left(25,27 \mathrm{~g} \mathrm{~kg}^{-1}\right)$ (Fig. 1A). Estos valores de concentración están por encima de los reportados por Reyes et al. (2014) y Buczkowska et al. (2015).

El tipo Tabasco presentó la mayor concentración de $\mathrm{N}$ en hojas en el tratamiento T3 $\left(50,46 \mathrm{~g} \mathrm{~kg}^{-1}\right)$ y la menor en T1 (40,84 $\left.\mathrm{g} \mathrm{kg}^{-1}\right)$; en fruto, T4 (21,63 $\left.\mathrm{g} \mathrm{kg}^{-1}\right)$ y $\mathrm{T} 1\left(16,85 \mathrm{~g} \mathrm{~kg}^{-1}\right)$. Fósforo, la mayor concentración en T3 $\left(4,89 \mathrm{~g} \mathrm{~kg}^{-1}\right)$ y menor en T1 $\left(4,19 \mathrm{~g} \mathrm{~kg}^{-1}\right)$; en fruto, T1 $\left(3,43 \mathrm{~g} \mathrm{~kg}^{-1}\right)$ y T4 $\left(2,84 \mathrm{~g} \mathrm{~kg}^{-1}\right)$ respectivamente. Potasio, con la mayor concentración en T4 $\left(42,82 \mathrm{~g} \mathrm{~kg}^{-1}\right)$ y menor en el T1 $\left(36,15 \mathrm{~g} \mathrm{~kg}^{-1}\right)$; en fruto, T4 (26,76 g $\mathrm{kg}^{-1}$ ) y T2 (24,42 $\mathrm{g} \mathrm{kg}^{-1}$ ) (figura 1B); estos valores de concentración de acuerdo a García et al. (2007) son superiores para $\mathrm{N}$ y bajos en el caso de P y K.

Los dos tipos de materiales mostraron igual comportamiento en el orden de concentración de nutrientes en hoja: $\mathrm{N}>\mathrm{K}>\mathrm{P}$ y en fruto: $\mathrm{K}>\mathrm{N}>\mathrm{P}$, como lo sugieren Constantino et al. (2008) y Azofeifa y Moreira (2005). Así, la mayor concentración de N se encontró en hojas y la mayor concentración de $\mathrm{K}$ en frutos, y

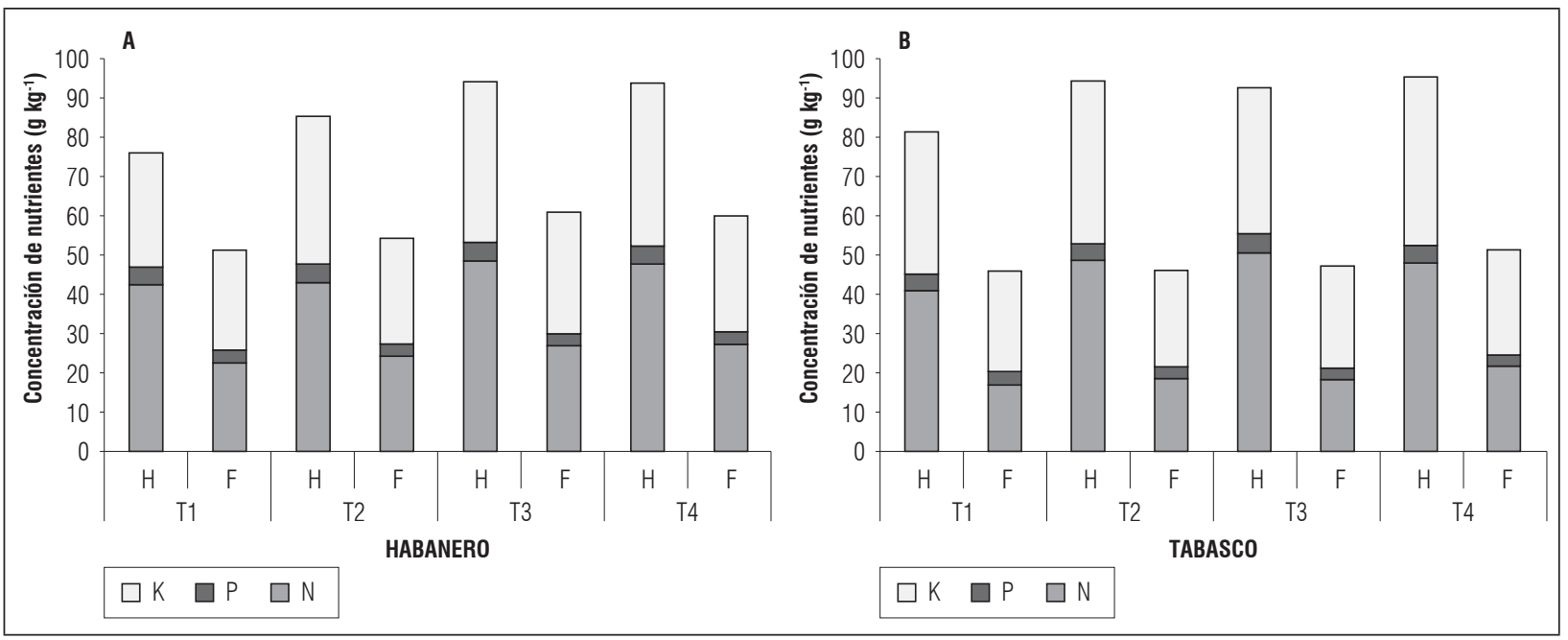

Figura 1. Concentración de nitrógeno, fósforo, potasio en hoja (H) y fruto (F) para dos tipos de material: (A) Habanero y (B) Tabasco. 
ambos coinciden en la menor concentración de P. Sin embargo, es generalizada la mayor concentración de N-P-K en hojas en comparación con los frutos.

\section{Rendimiento en ají tipo Habanero y Tabasco}

El rendimiento mostró diferencias altamente significativas $(P \leq 0,01)$ por efecto del tipo de material y tratamiento (Fig. 2); así, el mayor rendimiento se obtuvo en el tratamiento T4 (Habanero $23.807 \mathrm{~kg} \mathrm{ha}^{-1}$ y Tabasco $10.195 \mathrm{~kg} \mathrm{ha}^{-1}$ ), comportamiento normal dado que a mayor cantidad de $\mathrm{N}$ aplicado existe un mayor efecto sobre el rendimiento (Ayodele et al., 2015; Ortas, 2013); y el menor rendimiento en el tratamiento T1 (Habanero $12384 \mathrm{~kg} \mathrm{ha}^{-1}$ y Tabasco 4
$895 \mathrm{~kg} \mathrm{ha}^{-1}$ ), siendo mayor en Habanero con respecto a Tabasco. Estos valores de rendimiento se consideran bajos al compararlos con los reportados por Reyes et al. (2014). Sin embargo, comparados con Pardey et al. (2009) se consideran altos, coincidiendo en rendimientos superiores para ají tipo Habanero.

La relación entre la concentración de nutrientes en la planta (hoja y fruto) y el rendimiento, mostró que a mayor concentración de nutrientes en hoja y fruto coincide con mayor rendimiento, similares resultados encontraron Constantino et al. (2008) (Fig. 2); para Habanero en el tratamiento de mayor rendimiento T4, en hoja (Fig. 2A) hubo mayor concentración de $\mathrm{N}\left(47,36 \mathrm{~g} \mathrm{~kg}^{-1}\right)$, seguido de $\mathrm{K}\left(41,18 \mathrm{~g} \mathrm{~kg}^{-1}\right)$ y P $(4,56$ $\mathrm{g} \mathrm{kg}^{-1}$ ), y en fruto (Fig. 2C) se observa una mayor
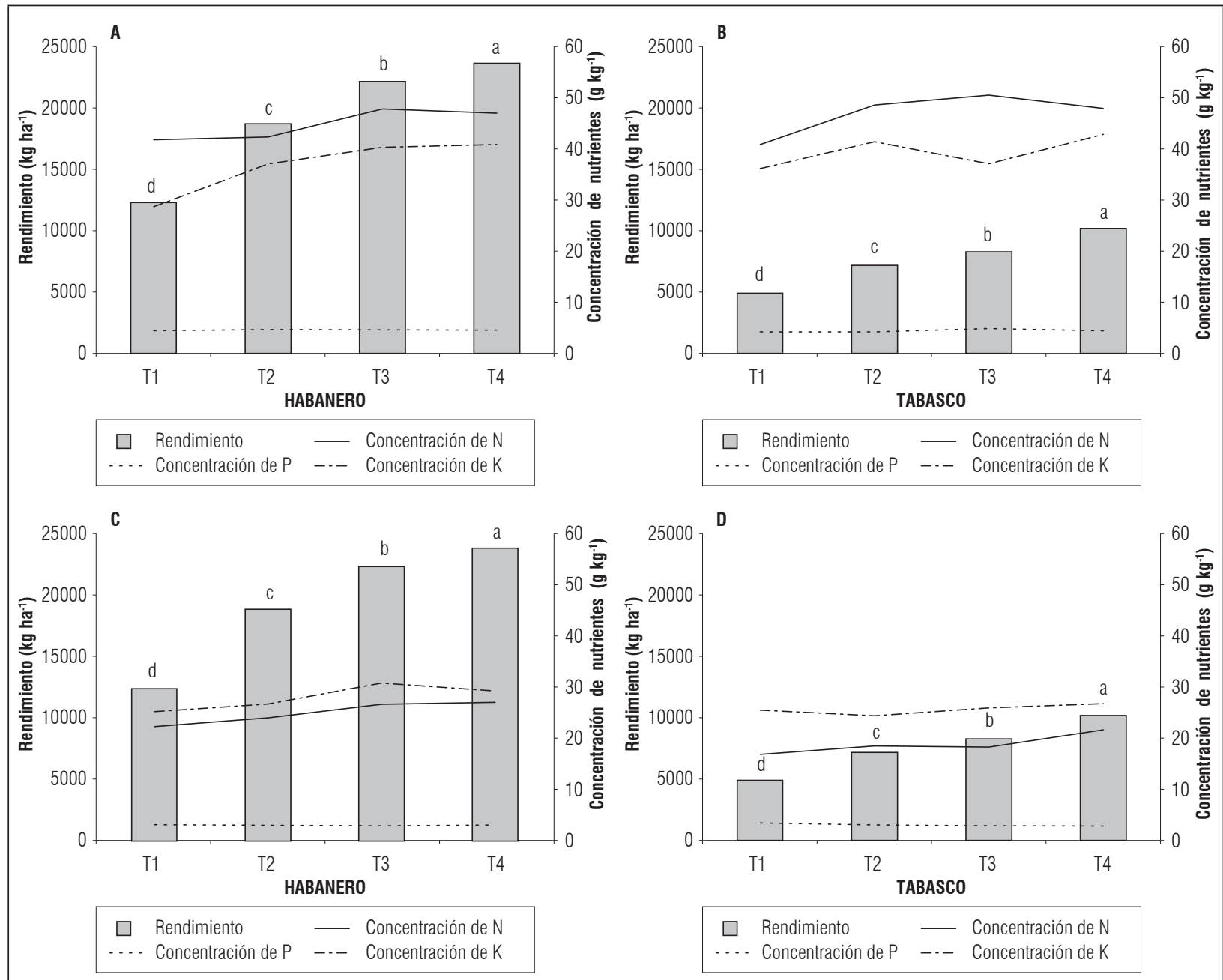

Figura 2. Relación de la concentración de nutrientes en hoja $(A$ y $B$ ) y en fruto (C y D) con respecto al rendimiento para Habanero y Tabasco. Promedios del rendimiento, con letras distintas, indican diferencia significativa según la prueba de Tukey $(P \leq 0,05)$. 
concentración de K (29,28 $\left.\mathrm{g} \mathrm{kg}^{-1}\right)$, seguido de $\mathrm{N}(27,09$ $\left.\mathrm{g} \mathrm{kg}^{-1}\right)$ y por último $\mathrm{P}\left(3,15 \mathrm{~g} \mathrm{~kg}^{-1}\right)$; el tipo Tabasco mostró en hoja (Fig. 2B) mayor concentración de $\mathrm{N}$ $\left(47,92 \mathrm{~g} \mathrm{~kg}^{-1}\right)$, seguido $\mathrm{K}\left(42,82 \mathrm{~g} \mathrm{~kg}^{-1}\right)$ y P $\left(4,43 \mathrm{~g} \mathrm{~kg}^{-1}\right)$, y en fruto (Fig. 2D) mayor concentración de K $(26,76$ $\left.\mathrm{g} \mathrm{kg}^{-1}\right), \mathrm{N}\left(21,63 \mathrm{~g} \mathrm{~kg}^{-1}\right)$ y $\mathrm{P}\left(2,84 \mathrm{~g} \mathrm{~kg}^{-1}\right)$.

El tipo de material Habanero mostró mayor rendimiento con respecto al tipo Tabasco; así las cosas, a mayor rendimiento mayor extracción de nutrientes, como lo sugieren Inzunza et al. (2010). En este sentido, el ají Habanero extrajo mayor cantidad de N-P-K para producir $1.000 \mathrm{~kg}$ de fruto fresco por hectárea comparado con Tabasco, mostrando diferencias altamente significativas $(P \leq 0,01)$ por efecto del tipo de material (Tab. 2).
Tabla 2. Extracción de nutrientes en $\mathrm{kg} \mathrm{ha}^{-1}$ por tonelada de fruto fresco.

\begin{tabular}{|c|c|c|c|}
\hline Tipo de material & $\mathrm{N}$ & $\mathrm{P}$ & $\mathrm{K}$ \\
\hline Habanero & $27,09 \mathrm{a}$ & $3,15 \mathrm{a}$ & $29,28 \mathrm{a}$ \\
\hline Tabasco & $21,63 \mathrm{~b}$ & $2,84 \mathrm{~b}$ & $26,76 \mathrm{~b}$ \\
\hline
\end{tabular}

Promedios con letras distintas, en la misma columna, indican diferencia significativa según la prueba de Tukey $(P \leq 0,05)$.

En términos porcentuales y de mayor a menor, la extracción de K-N-P para Habanero: 49,19; 45,5 y $5,29 \%$, respectivamente; y para Tabasco, $52,24 \% \mathrm{~K}$, $42,22 \%$ N y $5,54 \%$ P. Sin embargo, además de estos nutrientes la planta también extrae otros, tales como Mg, Ca, S, Fe, Mn, Zn, B, Cu y Na; en este sentido,

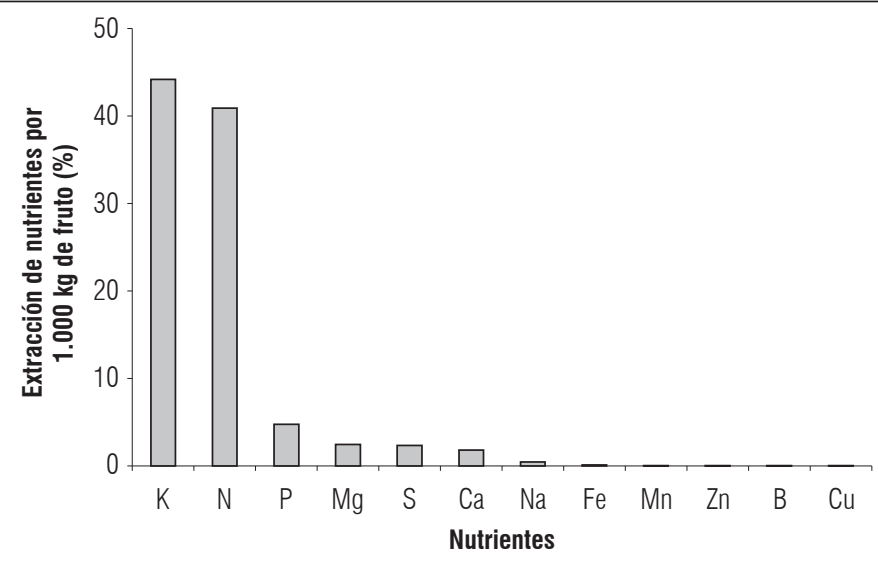

Figura 3. Extracción porcentual para 12 nutrientes en ají Habanero.

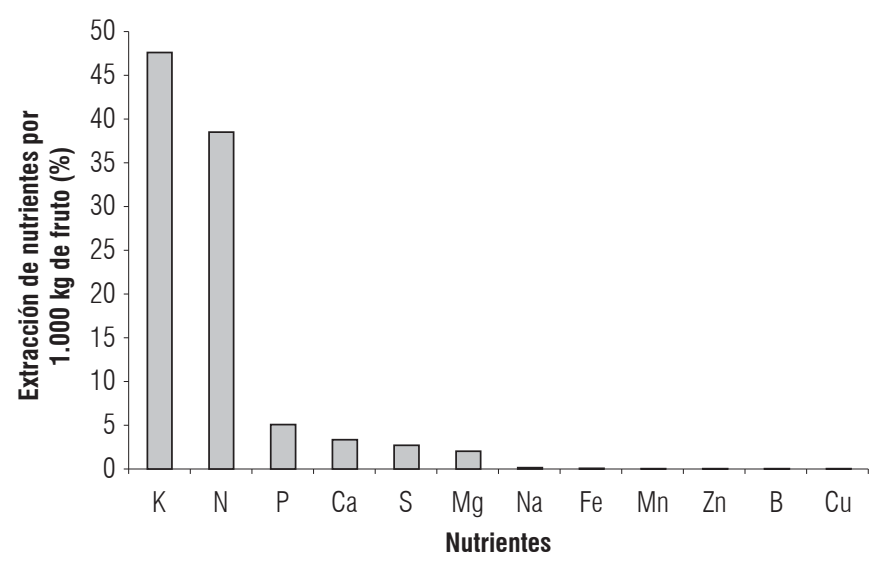

Figura 4. Extracción porcentual para 12 nutrientes en ají Tabasco. 
el orden de extracción para producir $1.000 \mathrm{~kg}$ de fruto fresco de ají incluyendo todos los nutrientes en porcentajes de mayor a menor en Habanero: K (44,25\%), N (40,95\%), P (4,76\%), Mg (2,46\%), S (2,34\%), Ca $(1,81 \%), \mathrm{Na}(0,44 \%), \mathrm{Fe}(0,08 \%), \mathrm{Mn}(0,02 \%), \mathrm{Zn}$ $(0,02 \%), \mathrm{B}(0,01 \%)$ y Cu $(0,01 \%)$ (Fig. 3$)$, presentando preferencia por $\mathrm{Mg}$ en lugar de $\mathrm{Ca}$, en contraste con el tipo de material Tabasco.

El orden de extracción para Tabasco de mayor a menor fue: K (47,88\%), N (38,71\%), P (5,08\%), Ca (3,35\%), $\mathrm{S}(2,71 \%), \mathrm{Mg}(2,02 \%), \mathrm{Na}(0,12 \%), \mathrm{Fe}(0,07 \%), \mathrm{Mn}$ $(0,02 \%), \mathrm{Zn}(0,02 \%), \mathrm{B}(0,02 \%)$ y Cu $(0,01 \%)$ (Fig. 4$)$, presentando preferencia por $\mathrm{Ca}$ en lugar de $\mathrm{Mg}$, en contraste con el material Habanero.

Es interesante la similitud en extracción que presentan los dos tipos de materiales mostrando el mismo orden de extracción para los nutrientes $\mathrm{K}>\mathrm{N}>\mathrm{P}>\mathrm{S}>$ $\mathrm{Na}>\mathrm{Fe}>\mathrm{Mn}>\mathrm{Zn}>\mathrm{B}>\mathrm{Cu}$, coincidiendo con el orden de extracción de nutrimentos propuesto por Azofeifa y Moreira (2005), sin embargo, difieren entre materiales en la cantidad extraída de nutrientes y su relación con el rendimiento.

\section{CONCLUSIONES}

Los tipos de material, Habanero y Tabasco, muestran un comportamiento diferencial al ser sometidos a igual plan de fertilización, tanto en la extracción en fruto y distribución de nutrientes en hoja y fruto, como en rendimiento. En este sentido, la dosis de $300 \mathrm{~kg} \mathrm{ha}^{-1}$ de N, $200 \mathrm{~kg} \mathrm{ha}^{-1} \mathrm{P}_{2} \mathrm{O}_{5}$ y $600 \mathrm{~kg} \mathrm{ha}^{-1} \mathrm{~K}_{2} \mathrm{O}$ contribuyen a un mayor rendimiento en Habanero y Tabasco, sin embargo, Habanero presentó mayor rendimiento, composición de nutrientes en hojas y fruto, y extracción en fruto fresco, lo cual evidencia la influencia del genotipo.

La especie C. chinense (Habanero) extrajo mayor cantidad de $\mathrm{kg} \mathrm{ha}^{-1}$ de N-P-K para producir $1.000 \mathrm{~kg}$ de fruto fresco con respecto $C$. frutescens (Tabasco), lo cual sugiere que al producir mayor cantidad de $\mathrm{kg}$ de fruto fresco se aumentará la extracción de nutrientes, en este sentido, se debe regresar al suelo esa misma cantidad para compensar la pérdida por extracción de nutrientes, la cual es diferente para cada especie. Así mismo, el contenido de nutrientes en hoja permitirá al agricultor hacer correcciones nutricionales que garanticen rendimientos de $23.807 \mathrm{~kg} \mathrm{ha}^{-1}$ y $10.195 \mathrm{~kg}$ ha ${ }^{-1}$ para Habanero y Tabasco respectivamente, contribuyendo a realizar un apropiado manejo nutricional por genotipo de ají.

\section{REFERENCIAS BIBLIOGRÁFICAS}

Alejo, S.G., E.G. Luna, H.R. Sánchez, P.E. Salcedo, P.J.D. García y M.V.M. Jiménez. 2015. Determination of the nitrogen requirement for habanero pepper (Capsicum chinense Jacq.) México. Rev. Chapingo Serie Hortic. 21(3), 215-221. Doi: 10.5154/r.rchsh.2014.04.015

Ayodele, O.J, E.O. Alabi y M. Aluko. 2015. Nitrogen fertilizer effects on growth, yield and chemical composition of hot pepper (Rodo). Int. J. Agr. Crop Sci. 8 (5), $666-673$

Azofeifa A. y M.A. Moreira. 2005. Absorción y distribución de nutrimentos en plantas de chile dulce (Capsicum annuum CV. UCR 589) en Alajuela, Costa Rica. Agron. Costarr. 29(1), 77-84

Bosland, P.W. y E.J. Votaba. 2012. Pepper: Vegetable and spice capsicums. $2^{\text {nd }}$ ed. CABI Publishing, Wallingford, UK. Doi: 10.1079/9781845938253.0000

Buczkowska, H., Z. Michałojć, J. Konopińska y P. Kowalik. 2015. Content of macroand microelements in sweet pepper fruits depending on foliar feeding with calcium. J. Elem. 20(2), 261-272. Doi: 10.5601/ jelem.2014.19.3.712

Constantino M.R., A.J.D. Gómez, S.V. Álvarez, G.E, Huerta y E. Barba. 2008. Effect of inoculation with rhizobacteria and arbuscular mycorrhizal fungi on growth and yield of Capsicum chinense Jacquin. J. Agric. Rural Develop. Trop. Subtrop. 109, 169-180.

García H.J.L., C.R. D. Valdez, V.R. Servín, D.E. Troyo, A.B. Murillo, P.E.O. Rueda, O.J.C. Rodríguez y O.R. Magallanes. 2007. Interacciones nutrimentales y normas de diagnóstico de nutrimento compuesto en un cultivar semidomesticado de Capsicum frutescens. Rev. Chapingo Ser. Hortic. 13(2), 133-140. Doi: 10.5154/r. rchsh.2007.01.004

Inzunza, I.M.A., V.C.M. Magdalena, V.E.A. Catalán y L.A. Román. 2010. Extracción de nutrientes y producción de chile jalapeño bajo acolchado plástico y niveles de riego. Terra Latinoam. 28(3), 211-218.

Malavolta, E., G.C. Vitti y S.A. de Oliveira. 1997. Avaliação do estado nutricional de plantas: Princípios e aplicações. Potafos. $2^{\text {a }}$ ed. Piracicaba, Brasil.

Marschner, H. 1995. Mineral nutrition of higher plants. Academic Press. $2^{\text {nd }}$ ed. London, UK.

Mckean, S.J. 1993. Manual de análisis de suelos y tejido vegetal: una guía teórica y práctica de metodologías. Documento de trabajo No.129. Laboratorio de Servicios Analíticos. Centro Internacional de Agricultura Tropical (CIAT).Cali, Colombia.

Nieves, G.F., S.G. Alejo, E.G. Luna, F.C. Lemus, L.P. Juárez y P.E. Salcedo. 2015. Extracción y requerimiento de fósforo en chile habanero (Capsicum chinense Jacq.) 'Big Brother'. Interciencia 40(4), 282-286. 
Noh, M.J., G.L. Borges y F.M. Soria. 2010. Composición nutrimental de biomasa y tejidos conductores en chile habanero (Capsicum chinense Jacq.). Trop. Subtrop. Agroecosyst. 12, 219-228.

Ortas, I. 2013. Influences of nitrogen and potassium fertilizer rates on pepper and tomato yield and nutrient uptake under field conditions. Sci. Res. Essays. 8(23), 1048-1055. Doi: 10.5897/SRE11. 579

Pardey, R.K., D.M.A. García y C.F.A. Vallejo. 2009. Evaluación agronómica de accesiones de Capsicum del banco de germoplasma de la Universidad Nacional de Colombia sede Palmira. Acta Agron. 58(1), 23-28.

Puentes, P.Y.J., F.J.C. Menjivar y H.F. Aranzazu. 2016. Concentración de nutrientes en hojas, una herramienta para el diagnóstico nutricional en cacao. Costa Rica. Agron. Mesoam. 27(2), 329-336. Doi:10.15517/ am.v27i2.19728

Quintal, O.W.C., G.A. Pérez, M.L. Latournerie, L.C. May, S.E. Ruiz y C.A.J. Martínez. 2012. Uso de agua, potencial hídrico y rendimiento de chile habanero (Capsicum chinense Jacq.). Rev. Fitotec. Mex. 35 (2), 155-160.

Reyes, R.A., A.M. López, S.E. Ruiz, M.L. Latournerie, G.A. Pérez, C.M.G. Lozano y L.M.J. Zavala. 2014.
Efectividad de inoculantes microbianos en el crecimiento y productividad de chile habanero (Capsicum chinense Jacq.). Agrociencia 48, 285-294.

Romero, L.M.P., P.Y.J. Puentes y F.J.C. Menjivar. 2016. Eficiencia de uso de nutrientes en ají tabasco (Capsicum frutescens L.) y habanero (Capsicum chinense Jacq). Rev. Investig. Agr. Ambient. 7(2), 121-127. Doi: 10.22490/21456453.1562

Salazar, J.F.I. y L.P. Juárez. 2013. Requerimiento macronutrimental en plantas de chile (Capsicum annuum L.). Rev. BioCiencias 2(2), 27-34.

Salisbury, F. B. y Ross, C.W. 2000. Fisiología de las plantas. Ed. Paraninfo Thomson Learning, Madrid, España.

Vallejo, C.F.A. y S.E.I. Estrada. 2004. Producción de hortalizas de clima cálido. Universidad Nacional de Colombia. Palmira, Colombia.

Zayed, M.S., M.K.K. Hassanein, H.E. Nahed y M.M.F. Abdallah. 2013. Productivity of pepper crop (Capsicum annuum L.). as affected by organic fertilizer, soil solarization, and endomycorrhizae. Ann. Agric. Sci. 58(2), 131-137. Doi: 10.1016/j.aoas.2013.07.011 